\title{
The complete mitochondrial genomes of two rice planthoppers, Nilaparvata lugens and Laodelphax striatellus: conserved genome rearrangement in Delphacidae and discovery of new characteristics of atp8 and tRNA genes
}

Kai-Jun Zhang ${ }^{1}$, Wen-Chao Zhu' ${ }^{1}$, Xia Rong ${ }^{1}$, Yan-Kai Zhang ${ }^{1}$, Xiu-Lei Ding ${ }^{1}$, Jing Liu', Da-Song Chen ${ }^{1}$, Yu Du and Xiao-Yue Hong ${ }^{1 *}$

\begin{abstract}
Background: Nilaparvata lugens (the brown planthopper, BPH) and Laodelphax striatellus (the small brown planthopper, SBPH) are two of the most important pests of rice. Up to now, there was only one mitochondrial genome of rice planthopper has been sequenced and very few dependable information of mitochondria could be used for research on population genetics, phylogeographics and phylogenetic evolution of these pests. To get more valuable information from the mitochondria, we sequenced the complete mitochondrial genomes of BPH and SBPH. These two planthoppers were infected with two different functional Wolbachia (intracellular endosymbiont) strains (wLug and wStri). Since both mitochondria and Wolbachia are transmitted by cytoplasmic inheritance and it was difficult to separate them when purified the Wolbachia particles, concomitantly sequencing the genome of Wolbachia using next generation sequencing method, we also got nearly complete mitochondrial genome sequences of these two rice planthoppers. After gap closing, we present high quality and reliable complete mitochondrial genomes of these two planthoppers.

Results: The mitogenomes of N. lugens (BPH) and L. striatellus (SBPH) are 17, 619 bp and 16, 431 bp long with A + T contents of $76.95 \%$ and $77.17 \%$, respectively. Both species have typical circular mitochondrial genomes that encode the complete set of 37 genes which are usually found in metazoans. However, the BPH mitogenome also possesses two additional copies of the trnC gene. In both mitochondrial genomes, the lengths of the atp8 gene were conspicuously shorter than that of all other known insect mitochondrial genomes (99 bp for BPH, 102 bp for SBPH). That two rearrangement regions ( $\operatorname{trn} C-\operatorname{trn} W$ and nad6-trnP-trnT) of mitochondrial genomes differing from other known insect were found in these two distantly related planthoppers revealed that the gene order of mitochondria might be conservative in Delphacidae. The large non-coding fragment (the A+T-rich region) putatively corresponding responsible for the control of replication and transcription of mitochondria contained a variable number of tandem repeats (VNTRs) block in different natural individuals of these two planthoppers. Comparison with a previously sequenced individual of SBPH revealed that the mitochondrial genetic variation within a species exists not only in the sequence and secondary structure of genes, but also in the gene order (the different location of $\mathrm{trnH}$ gene).

\footnotetext{
* Correspondence: xyhong@njau.edu.cn

'Department of Entomology, Nanjing Agricultural University, Nanjing

210095, China

Full list of author information is available at the end of the article
} 
(Continued from previous page)

Conclusion: The mitochondrial genome arrangement pattern found in planthoppers was involved in rearrangements of both tRNA genes and protein-coding genes (PCGs). Different species from different genera of Delphacidae possessing the same mitochondrial gene rearrangement suggests that gene rearrangements of mitochondrial genome probably occurred before the differentiation of this family. After comparatively analyzing the gene order of different species of Hemiptera, we propose that except for some specific taxonomical group (e.g. the whiteflies) the gene order might have diversified in family level of this order. The VNTRs detected in the control region might provide additional genetic markers for studying population genetics, individual difference and phylogeographics of planthoppers.

\section{Background}

The mitochondrial genome is the most commonly used molecular marker for phylogenetic studies, population genetics and dynamics, phylogeography [1] and even for mitochondrial related insecticide/acaricide resistance [2]. It also can provide very favourable information at the genome level, such as the relative positions of different genes [3-7], strand asymmetry in nucleotide composition [8] and evolutionary patterns of the control region [9]. Furthermore, some special structural features such as minicircles could exist in some specified groups of insects $[10,11]$.

The order Hemiptera is the largest group of the hemimetabolous insects [12] and includes three phylogenetically controversial suborders: Auchenorrhyncha, Sternorrhyncha, and Heteroptera [13]. The complete mitochondrial genome sequences have been determined for more than 300 species of insects so far. Within Hemiptera, although 47 complete or nearly complete mitochondrial genomes are available at NCBI (as of October 1, 2012), still very few species in some groups, especially in the suborder Auchenorrhyncha (7 species) and Sternorrhyncha (9 species) had been sequenced. In the Delphacidae, only one species has had its mitochondrial genome sequenced so far [14]. The phylogenetic relationships of this order at the superfamily and suborder levels are still controversial [14-18]. To solve these controversial phylogenetic problems in this order requires more information from mitochondrial genomes and even from nuclear genes [19].

The brown planthopper (BPH), Nilaparvata lugens and the small brown planthopper (SBPH), Laodelphax striatellus (Hemiptera: Delphacidae) are two of the most important pests in rice fields of many Asian countries. By transmitting virus diseases [20] and causing directly feeding damage, these two planthoppers cause serious yield losses of rice and economic loss for taking control measures each year [21]. These two planthoppers were also infected with the most widespread endosymbiont Wolbachia [22] which can manipulate the reproduction and affect the fitness of its host [23,24]. While sequencing the genomes of two different phenotype-inducing Wolbachia strains, we also obtained nearly the complete mitochondrial genomes of BPH and SBPH. The Wolbachia strain wStri infecting SBPH can induce complete Cytoplasmic Incompatibility $(\mathrm{CI})$, a reproductive modification that typically results in embryonic lethality between crosses of infected males and uninfected females. On the other hand, the Wolbachia strain wLug infecting $\mathrm{BPH}$ cannot induce $\mathrm{CI}$ at all.

After gap-closing, the complete mitochondrial genomes of these two most important rice piercing and sucking type pests, BPH and SBPH were determined. We analyzed the nucleotide composition, codon usage and genomic rearrangement of these two mitochondrial genomes and compared them with mitochondrial genomes of other hemipteran species. Some special characteristics of mitochondria of these two planthoppers (for example, the variable number of tandem repeats (VNTRs) in 'A + T' rich regions; location of trnH gene) were verified using natural populations. In addition, comparing mitochondrial genome of SBPH sequenced in this study with a previously sequenced individual revealed a remarkable variability between these two individuals.

\section{Result and discussion}

Genome organization, structure and composition

One scaffold containing 4 contigs of $N$. lugens $(\mathrm{BPH})$ mtDNA and another scaffold containing 3 contigs of $L$. striatellus $(\mathrm{SBPH})$ were identified in the assemblage of the $w$ Lug and $w$ Stri genome (Figure 1). After gap closing, the assembled result showed that the mitochondrial genomes of $N$. lugens and L. striatellus are typical circular DNA molecules with $17,619 \mathrm{bp}$ and $16,431 \mathrm{bp}$ in length, respectively. Both mitochondrial genomes include the entire set of 37 genes usually present in animal mtDNAs [1], i.e. 13 PCGs, 22 tRNA genes, and 2 ribosomal genes (Figure 2). Except for the whitefly Trialeurodes vaporariorum, the mitochondrial genome of $\mathrm{BPH}$ is the longest in Hemiptera (the longest in suborders Auchenorrhyncha and Heteroptera) which were reported so far. The unusual length of the mitochondrial genome in $N$. lugens is partly due to a long putative control region and a long repeat region containing 


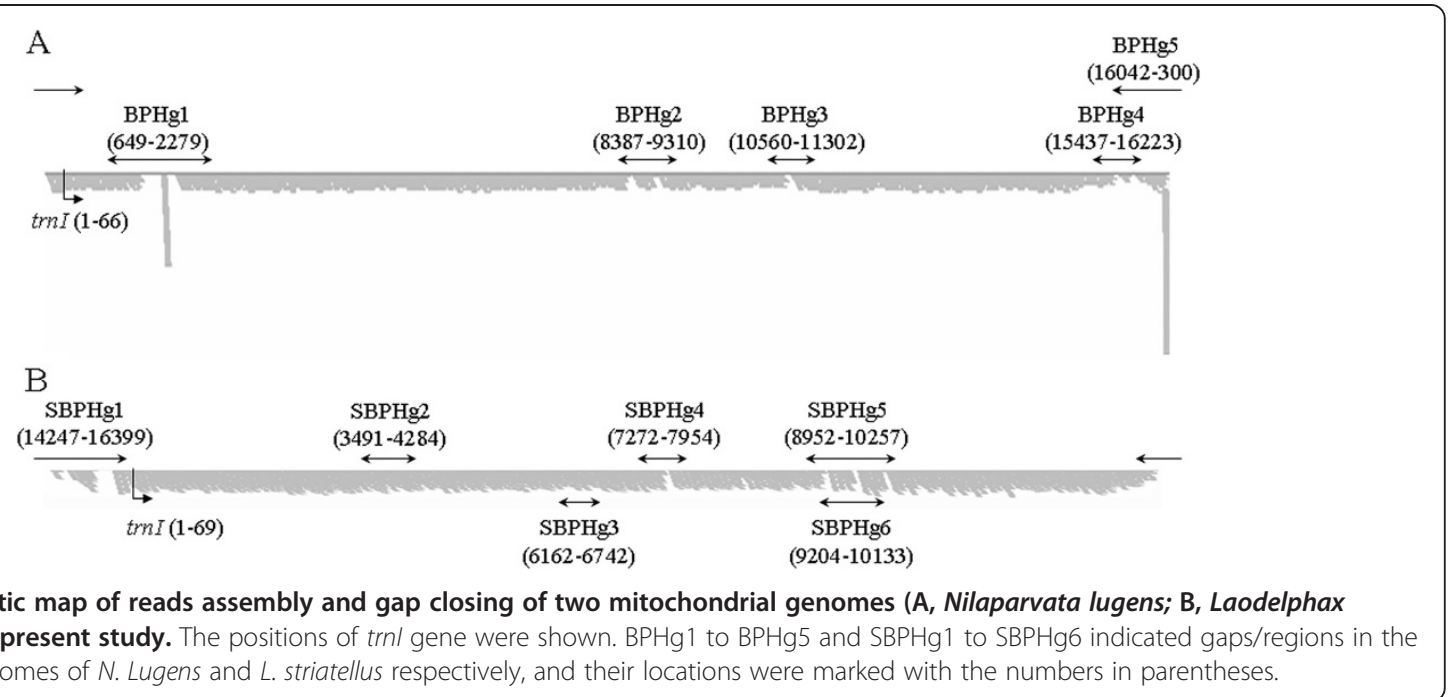

three repeats of $\operatorname{trn} C$ gene (Figure 2, Additional file 1: Table S1). The repeat characteristic is supported by the depth of sequencing this region compared with other normal regions (Figure 1). However, it is unclear whether the gene duplication is correlated with function variation. Other species with duplicated genes in the mitochondrial genomes include Leptotrombidium pallidum [25] and Metaseiulus occidentalis [26].
All of the tRNA genes have the typical cloverleaf secondary structure, except for trnS1 (AGN) of BPH, trnS2 (UCN) of SBPH which harbor a simple loop in the dihydrouridine (DHU) arm and trnH of SBPH could have a loop-stem structure like trnT of BPH (Additional file 2: Figure S1, Additional file 3: Figure S2). The dihydrouridine (DHU) arm of trnS1 (AGN) that forms a loop is found in many Hemiptera [17,18,27-30] and other metazoans [31].
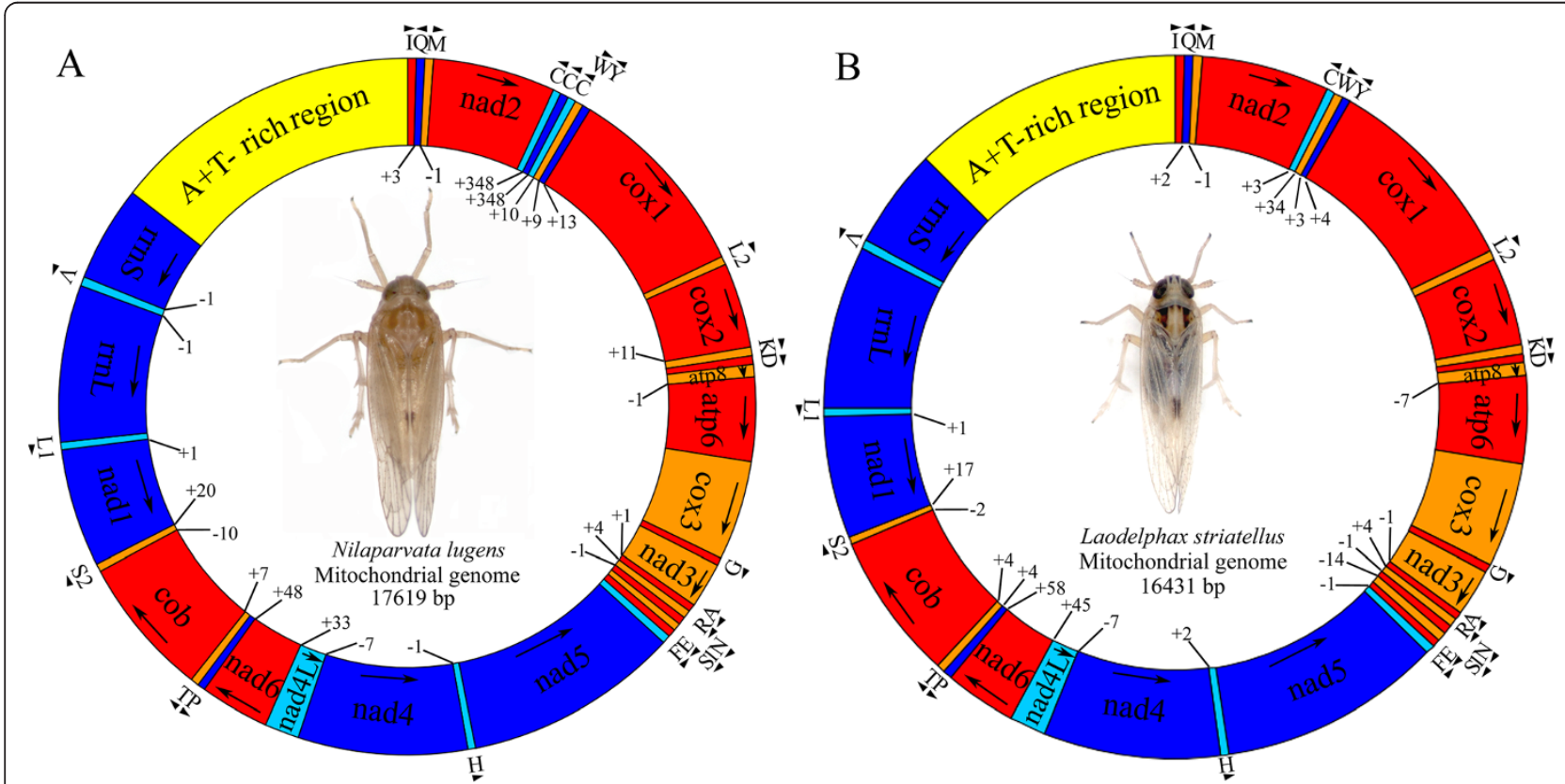

Figure 2 Map of the mitochondrial genome of Nilaparvata lugens (A) and Laodelphax striatellus (B). Genes coded in the J-strand (clockwise) are red or orange colored. Genes coded in the N-strand (counterclockwise) are blue or cyan colored. AT region (putative control region) is given in yellow colored. Gene names are the standard abbreviations used in this paper. Twenty two tRNA genes are indicated by the single letter IUPAC-IUB abbreviation for their corresponding amino acid in the draw. Arrows and arrowheads show the direction of the transcription of each gene. Numbers at gene junctions indicate the length of small non-coding regions where negative numbers indicate overlap between genes. 
For $\mathrm{SBPH}$, the secondary structure in the dihydrouridine (DHU) arm in the trnS1 (AGN) has a completed loopstem structure while that of trnS2 (UCN) has a loop. In contrast, the trnS1 (AGN) has a loop and trnS2 (UCN) has a completed loop-stem structure in previously reported SBPH [14]. Furthermore, it is similar that the $\operatorname{trnH}$ gene of SBPH which formed a weak loop-stem structure of the T-arm (TYC) in this study possessed a stable loopstem structural of the T-arm (TYC) in previous study [14].

The length of all tRNA genes ranged from $56 \mathrm{bp}$ for trnS2 (UCN) (SBPH) and 57 bp for trnS1 (AGN) (BPH) to $71 \mathrm{bp}$ for $\operatorname{trnK}(\mathrm{BPH}$ and $\mathrm{SBPH})$ and $\operatorname{trn} V(\mathrm{BPH})$ (Additional file 1: Table S1 and Additional file 4: Table S2). As in other hemipteran species, BPH and SBPH harbor two rRNA genes, $r r n S$ (748 bp for BPH and 747 bp for SBPH) and $r r n L$ (1,219 bp for both). They are located between trnL1 (CUN) and an A+T-rich region, separated by $\operatorname{trn} V$.

\section{Mitochondrial gene order in Hemiptera}

The mitochondrial gene orders of $\mathrm{BPH}$ and $\mathrm{SBPH}$ are significantly different from those of all other species of hemipteran insects. Two rearrangement regions $(\operatorname{trn} C$ $\operatorname{trn} W$ and nad6-trnP-trnT) associated with tRNAs and PCGs were found in both of BPH and SBPH (Figure 3). The same gene order was found in the white-backed planthopper (WBPH), Sogatella furcifera (unpublished data). But the most striking feature was the different locations of the $\operatorname{trnH}$ gene in two individuals of SBPH. The $\operatorname{trn} H$ gene is located between nad5 and nad4 genes in this study just as in all other hemipteran species sequenced so far, but Song and Liang (2009) previously reported that this tRNA gene is located between nad4L and nad6 genes (Figure 3) [14]. How could different gene orders appear in one species? Did the arrangement occur recently? We can not answer these questions yet.

However, the arrangement of these 37 genes, especially the arrangement of large genes that encode proteins and
rRNAs, is usually conserved in the suborders Auchenorrhyncha and Heteroptera, except for the uniqueheaded bug Stenopirates sp. [28]. In this study, two long intergenic regions in upstream and downstream of nad6 gene which contain a poly " $T$ " and poly " $\mathrm{A}$ " stretch might provide some clues of rearrangement trace. In addition to rearrangement events involving large genes, rearrangement of trnI and trnQ in Neuroctenus parus and the rearrangement of trnT and trnP in Physopelta gutta and Dysdercus cingulatus have also been reported [32]. Except for these 4 species of Heteroptera, 2 planthoppers of Delphacidae in Auchenorrhyncha and 6 species of Aleyrodidae in the suborder Sternorrhyncha [33], the gene order of mitochondrial genomes of all the other species (27 species (22 families) in Heteroptera, 3 species (2 families) of Sternorrhyncha, 6 species (6 families) of Auchenorrhyncha) were found to be consistent with the mitochondrial gene order of the putative ancestral insect (Figure 3, Additional file 5: Table S3). Considering the fact that some families in the same superfamily have different gene arrangements of mitochondrial genome (Three families, Issidae, Fulgoridae and Flatidae of superfamily Fulgoroidea have the same gene order of mitochondria as the proposed ancestral insect, but members of the family Delphacidae have a different one), we proposed that excluding the whiteflies, the arrangement of genes in the mitochondria could be considered as a factor in inferring phylogenetic relationships of different species at the family level of hemipteran insects. In any case, further genome sequencing is necessary to establish whether this feature is a mitochondrial signature of the whole order.

\section{Protein-coding genes (PCGs) and codon usage patterns}

The mtDNAs of BPH and SBPH contain the full set of PCGs usually presented in animal mtDNA. Except for the nad6 gene, PCGs are arranged along the genome according to the proposed standard order of Insects [1] (Figure 2). All PCGs have typical start codons. Both of

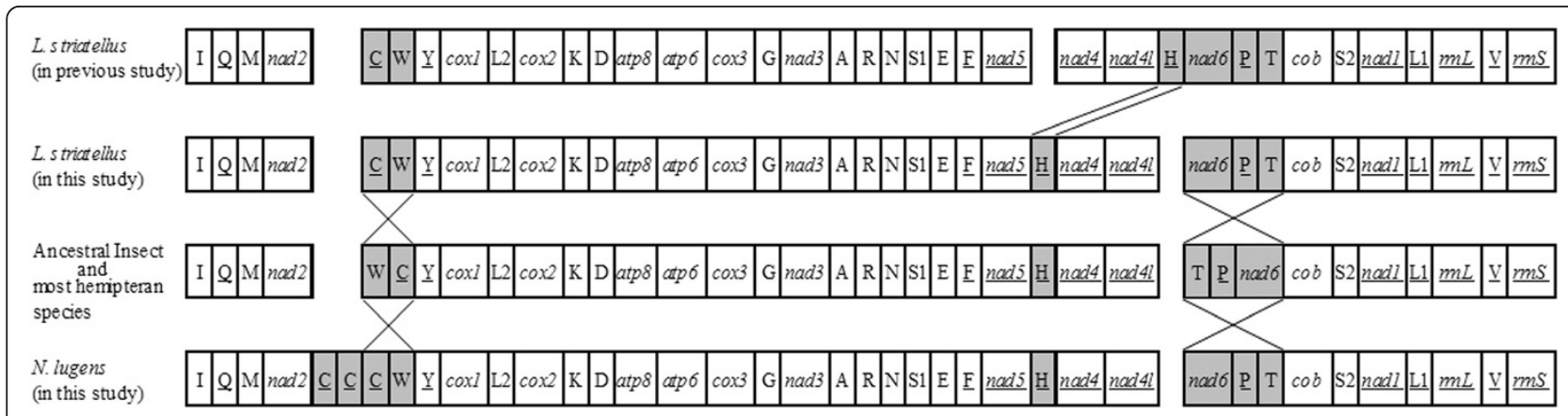

Figure 3 Schematic representation of mitochondrial gene arrangements in Nilaparvata lugens and Laodelphax striatellus. Underlined genes and tRNAs with letters below are encoded in the $\mathrm{N}$-strand. Shaded boxes indicate genes involving mitochondrial genome arrangement. Most of the currently determined hemipteran species have the ancestral gene order. Previously sequenced Laodelphax striatellus has a translocation of $\mathrm{trnH}$ gene. 
the putative start codons ATG and ATT are used in $\mathrm{BPH}$ and SBPH (Additional file 1: Table S1, Additional file 4: Table S2). Compared with $\mathrm{SBPH}, \mathrm{BPH}$ has one more kind of initiation codon ATA in nad3 and atp6, while ATA was not observed in SBPH. The cox1, cox3, atp6, and nad5 genes of these two mtDNAs have incomplete stop codons. For the cox2 gene, these two planthoppers have different stop codons, TAA for BPH and TAG for SBPH (Additional file 1: Table S1, Additional file 4: Table S2). The atp 8 and atp6 of $\mathrm{BPH}$ and $\mathrm{SBPH}$ are the only PCGs having different overlapping nucleotides (1 bp for $\mathrm{BPH}$, 7bp for $\mathrm{SBPH}$ ). This feature is common in hemipteran insects [17], and is similar to all lepidopteran mitochondrial genomes known [34] and many other animal mtDNAs [1].

It is worth mentioning that the lengths of atp 8 gene of planthoppers (99 bp for BPH, 102 bp for SBPH) were the shortest in insects known so far. In fact, the longest atp 8 gene (228 bp) was found in Bemisia tabaci (Hemiptera: Aleyrodidae) [34]. In almost all other insects, the length ranged from 138 bp (Hemiptera: Psyllidae: Pachypsylla venusta) [33] to 183 bp (Lepidoptera: Lymantriidae: Lymantria dispar) [35]. In most insects, atp8 gene had a length of about $160 \mathrm{bp}$. Taking into account the length of atp 8 gene of planthoppers discovered in this study, it seems that the length distribution of atp 8 gene in Hemiptera was wider (From 99 bp to $228 \mathrm{bp}$ ) than that of all other orders of insects reported so far. The sequence alignment of atp 8 gene with other insects revealed that nearly 60 bp length of sequence in the 3 'end of this gene was lost in these two planthoppers. Does the short length of this gene change the structure of its encoded protein? Does length change of this gene alter the functions or efficiency of this gene? These aspects are worth further studying.

The abundance of codon families and Relative Synonymous Codon Usage (RSCU) [36] in PCGs in BPH and SBPH mtDNAs are summarized in Figure 4. The total numbers of non-stop codons (CDs) used by these two analyzed mtDNAs of planthoppers are very similar, 3,608 for $\mathrm{BPH}$ and 3,613 for SBPH. The codon families exhibit a very similar behavior among these two species. The most frequently used amino acid is Leu (BPH: 14.86\%; SBPH: $15.42 \%)$, followed by Phe (BPH: 11.59\%; SBPH: $11.13 \%)$, Ile (BPH: 11.12\%; SBPH: 10.79\%), Ser (BPH: 11.06\%; SBPH: $10.27 \%)$ and Met (BPH: 7.43\%; SBPH: 6.70\%). The proportion of these five amino acids is more than $54 \%$. Two codons (GCG, CGC) were not observed in the PCGs (Figure 4). CGC codons were also absent in the PCGs in many other hemipteran species [32].

\section{Control region of mitochondrial genome of $\mathrm{BPH}$ and SBPH}

The control regions of $\mathrm{BPH}$ and SBPH mitogenomes are located at the conserved position between $r r n S$ and trnI-
trnQ-trnM gene cluster (Figure 2) with $79.3 \%$ and $83.2 \%$ $\mathrm{A}+\mathrm{T}$ content, respectively. The lengths of control regions of mitochondrial genome of $\mathrm{BPH}$ and $\mathrm{SBPH}$ were obvious different (BPH: 2429 bp; SBPH: 2042 bp). The near 390 bp length difference was contributed by the different numbers of repeat unit. The repeat region of mitochondrial genome of $\mathrm{BPH}$ contained 55 times repeat of unit "GGAAAAAATGTCACGTTTTT(C/T)", while similar repeat unit of SBPH "CACGATTTTTGG AAAAAATGT" showed 35 times repeat. But in repeat regions of both mitochondria contained incomplete repeat unit: the repeat 2 of $\mathrm{BPH}$ and the repeat 3, 14, 35 of SBPH were not complete. Within the same superfamily (Fulgoroidea) of these two planthoppers, the similar repeat units in control region had once reported in Geisha distinctissima [17] and Sivaloka damnosus [16], though the lengths of repeat regions were much shorter than that of SBPH and BPH. In contrast, a small number of long tandem repeats are commonly appeared in species of Heteroptera [18,28,32,37]. Actually, the variable number of tandem repeats (VNTRs) of mitochondrial genome of $\mathrm{BPH}$ and $\mathrm{SBPH}$ were discovered in natural populations (Figure 5). PCR amplification a fragment of mitochondrial genome containing the repeat regions of different individuals from three natural populations of each of these two planthopper species exhibited length polymorphism. The length polymorphism caused by variable repeat numbers was also confirmed by sequencing the repeat regions of several random selected individuals. Usually due to the presence of variable copy numbers of repetitive elements, the control region is considered as the source of size variation in the entire mitochondrial genome [38]. In this study, we revealed that the variable copy numbers of repetitive element could emerge in different individuals of planthoppers which were even collected from the same geographical population. This characteristic might provide additional genetic markers for studying population genetics, individual difference and phylogeographics of planthoppers.

In addition, both of these two mitochondrial genomes possess a long poly " $\mathrm{T}$ " stretch $(23 \mathrm{bp})$ in the A+T-rich region. This poly " $\mathrm{T}$ " structure might be conservative in Delphacidae and be responsible for the control of replication and transcription of mitochondria.

\section{Base composition and AT/GC-skew of mtDNA of Hemiptera}

The composition of the majority strand (J-strand) of SBPH mtDNA is $\mathrm{A}=7099$ (43.2\%), $\mathrm{T}=5581$ (33.97\%), $\mathrm{G}=1527$ (9.29\%) and $\mathrm{C}=2224$ (13.54\%). The composition of the $\mathrm{J}$ strand of $\mathrm{BPH}$ mtDNA is $\mathrm{A}=7393$ (41.96\%), $\mathrm{T}=6165$ (34.99\%), $\mathrm{G}=1661$ (9.43\%) and $\mathrm{C}=$ 2400 (13.62\%). Similarly to most hemipteran insects, the nucleotide compositions of the two planthopper mitogenomes are significantly biased toward A and $\mathrm{T}$ 


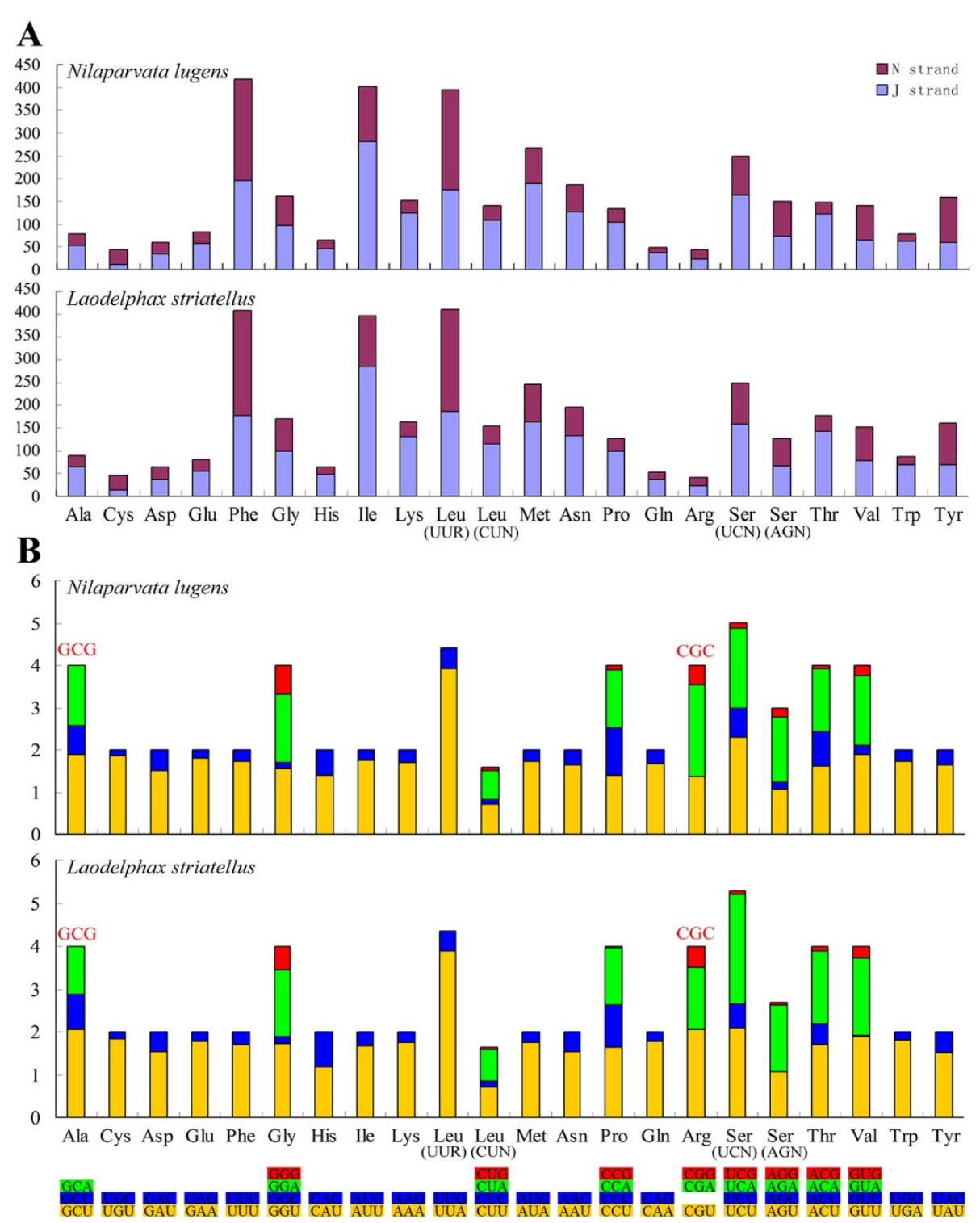

Figure 4 Codon usage pattern (A) and the relative synonymous codon usage (RSCU) (B) of Nilaparvata lugens and Laodelphax striatellus mitochondrial genomes. Numbers to the left refer to the total number of codons (A) and the RSCU value (B). Codon families are provided on the X-axis. Absent codons of the two mitogenomes are provided the top of columns.

(BPH: 76.95\%; SBPH: 77.17\%). Comparing with 48 mitochondrial genomes of hemipteran species, we found that there is considerable variation in base composition among different hemipteran species: ranging from $68.9 \%$ (Neuroctenus parus) to $86.3 \%$ (Aleurodicus dugesii). These two extreme $\mathrm{A}+\mathrm{T}$ content species were inferring gene arrangement $[32,33]$. But in different taxonomical groups of Hemiptera, the $\mathrm{A}+\mathrm{T} \%$ values show different ranges: Heteroptera (31 species, including 5 near complete mitochondrial genomes): $68.9-82.5 \%$, average: $74.7 \pm 2.95 \%$; Sternorrhyncha (9 species): $72-86.3 \%$, average: 78.79 $\pm 5.46 \%$; Auchenorrhyncha (8 species): 75.1-78.4\%, average: $76.86 \pm 0.95 \%$. The average $\mathrm{A}+\mathrm{T} \%$ value for all analyzed mtDNAs set is $75.83 \pm 3.67 \%$.

The hemipteran AT-skews vary from -0.1812 (Trialeurodes vaporariorum) to 0.2765 (Lycorma delicatula) with the $\mathrm{BPH}$ and SBPH mtDNA exhibiting a slight different AT-skew (0.0909 for BPH and 0.119171 for SBPH). The GC-skews of hemipteran species vary from -0.2827 (Acyr thosiphon pisum) to 0.2086 (Neomaskellia andropogonis) with the $\mathrm{BPH}$ and SBPH mtDNAs exhibiting very similar GC-skew ( -0.1826 for BPH and -0.1842 for SBPH). Similarly, the AT/GC-skew values exhibit different ranges among three suborders: AT-skew: Heteroptera, 0.0642 - 

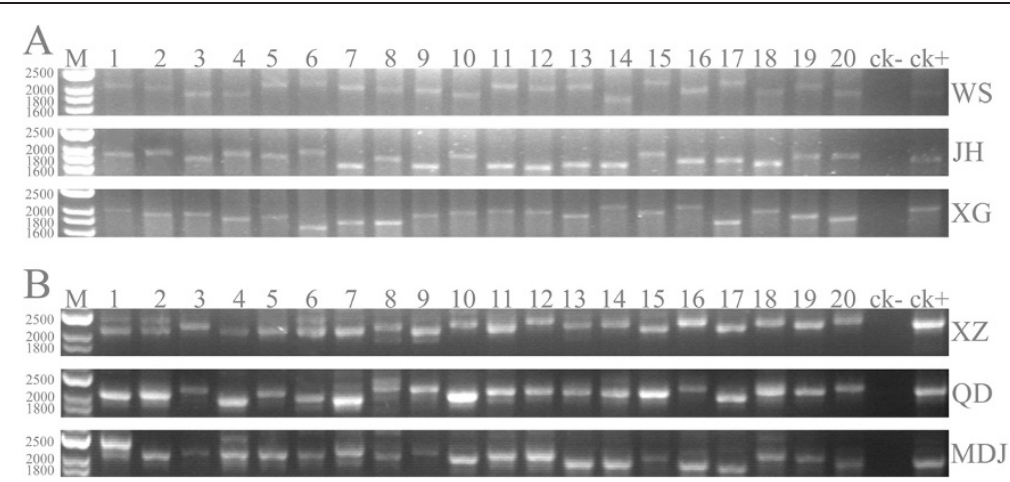

Figure 5 PCR assays the fragment length polymorphisms of control region of Nilaparvata lugens (A) and Laodelphax striatellus (B). M, molecular weight marker with sizes indicated on the left. The numbers above electropherogram indicated 20 individuals of each population were detected. The population codes (see Additional file 11: Table S7) are show on the right.

0.2181 , Sternorrhyncha, $-0.1812-0.0956$, Auchenorrhyncha, 0.0623 - T 0.2765; GC-skew: Heteroptera, -0.2656-(-0.0965), Sternorrhyncha, $-0.2810-0.2086$, Auchenorrhyncha, -0.282 7 - (-0.0783).

However, comparing the base compositions with the base skew values revealed that there is no correlation between the base compositions and the base skew values in any suborder of Hemiptera. For example, the $\mathrm{A}+\mathrm{T} \%$ values of different species of Auchenorrhyncha were much more similar than that of Heteroptera, but the range of AT/GC-skew values of this suborder was wider than Heteroptera. Even in the same suborder, the
AT/GC-skew values could show great difference among different groups. Compared with other three species (belong to two families) of Sternorrhyncha, all species of Aleyrodidae exhibit converse AT/GC-skew values (Figure 6) as described in previous study [33]. These characteristics indicate that except some specific groups (species in Aleyrodidae), the base compositions and base skew values of mitochondrial genomes of hemipteran insects might be similar in low level of taxa (e.g. in the level of family) other than in the level of suborder. Furthermore, the different base compositions and base skew values to some extent reflect different taxa of

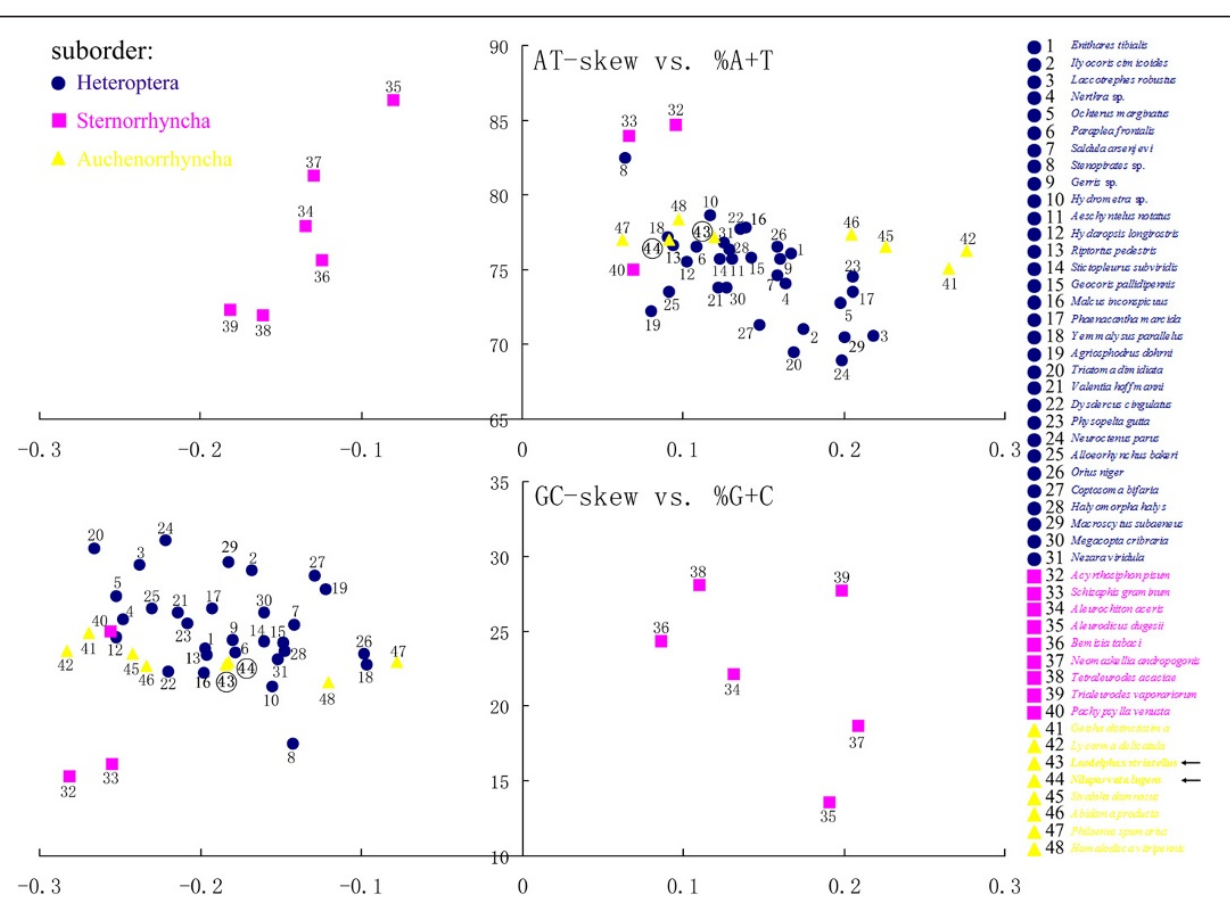

Figure 6 AT\% vs AT-skew and GC\% vs GC-skew in the $\mathbf{4 8}$ hemipteran mitochondrial genomes. Values are calculated on J-strands for full length of $m t$ genomes. The $X$-axis provides the skews values, while the $Y$ axis provides the $A+T / G+C$ values. Names of species are colored according to their taxonomic placement (see more information of these species in Additional file 5: Table S3). 
Hemiptera might have suffered different evolutionary selection pressures.

The mitochondrial genome is an effective data source for resolving deep-level phylogenetic problems [39], especially in intraordinal relationships, such as in Diptera [40], Hymenoptera [41], Orthoptera [42] and Hemiptera [27]. Phylogenic analysis based on all three codon positions or first and second codon positions of PCGs can get similar results of phylogenetic relationships of Hemiptera $[14,16]$. But based on the rRNA genes $[16]$ or on nuclear gene combination with mitochondrial data [15] generated a different phylogenetic relationship. However, when comparing $\mathrm{A}+\mathrm{T}$ content of all PCGs, $r r n L$ gene and $r r n S$ gene with the entire mitochondrial genome, PCGs show more significant positive correlation $\left(\mathrm{R}^{2}=\right.$ $0.9674)$ than $r r n L$ gene $\left(R^{2}=0.8873\right)$ and $r r n S$ gene $\left(\mathrm{R}^{2}=0.7189\right)$ (Additional file 6: Figure S3). The PCGs seem to better reflect the evolution of the entire mitochondrial genomes than the rRNA genes. Comparing with the nuclear data set, why the mitochondrial data set result in different phylogenies in Hemiptera? Actually, many factors can influence final tree [19]: the quality of the sequences and the alignment, the amount of phylogenetic information, compositional heterogeneity, unreasonable marker choices, the accuracy of the evolutionary model and the efficiency of the tree search algorithm used. In this study, the data set of hemipteran insects show apparent base compositional heterogeneity, especially in the suborder Sternorrhyncha and Auchenorrhyncha (Figure 4). Nonstationary sequence evolution leading to unequal nucleotide composition can cause inference methods to fail and phylogenies to be inaccurate in beetles [43]. Similarly, when we chose mitochondrial data to recover the phylogenies of Hemiptera, compositional heterogeneity of mitochondrial genomes among hemipteran species should also be considered.

\section{Comparative analysis of two mitochondrial genomes of L. striatellus}

The most obvious difference between the two mitochondrial genomes of L. striatellus was the location of the trnH: between nad5 and nad4 in this study just like the gene order of most hemipteran insects (Figure 3), while between nad4L and nad6 in previous study [14]. Amplification of the region encompassing the $\operatorname{trnH}$ gene with the primers SBPHg4F and SBPHg4R (Additional file 7: Table S4) produced a fragment (642 bp) which is composed of portion of the nad5 gene (482 bp), complete trn $H$ gene (62 bp), two intergenic nucleotides and a portion of nad4 gene (96 bp). All sequenced individuals (309 individuals from 15 locations) have an identical sequence of the $\operatorname{trn} H$ gene which was the same with that of the mitochondrial genome of SBPH sequenced in this study. Considering the low probability of many different sites in the $\operatorname{trn} H$ gene related sequences caused by PCR or sequencing, we still cannot exclude the possibility that some sporadic individuals of SBPH possessing the same location of $\operatorname{trnH}$ gene as previous study exist in some special regions in China. Anyway, according to the mutation sites (15 sites in nad5 gene, one site in the intergenic region between the $\operatorname{trnH}$ gene and the nad4 gene) of all sequences of the region compassing trnH gene, 16 haplotypes of the sequence were identified (Figure 7). The vast majority of individuals were belong to Haplo1 (178 individuals), Haplo2 (93 individuals) and Haplo3 (18 individuals) which were separated by two nonsynonymous nucleotide mutation at position 277 (amino acid substitution: M93L) and 391 (amino acid substitution: D131N) in nad5 gene. There are also 4 nonsynonymous nucleotide mutations found in Haplp4, 12, 13, 14 (Additional file 8: Table S5). The cotransmission of Wolbachia and mitochondria causes the bacteria to have an indirect impact on the DNA diversity of the mitochondria as a result of a selective sweep of the mitotype associated with abnormal production-induced Wolbachia infection [44-48]. Considering the nearly $100 \%$ infection rate of Wolbachia and the small diversity of mitochondria (less than 0.01) were observed in natural populations (Additional file 8: Table S5), we proposed that the invasion of Wolbachia in SBPH occurred recently. In Jingyuan populations, all 5 individuals of SBPH were not infected with Wolbachia but shared mitochondrial haplotypes with other populations suggested that the Wolbachia might be lost in this natural population.

Another significant difference of these two mitochondrial genomes of SBPH was the distinct lengths of four NADH dehydrogenase genes (nad2, nad4, nad5, nad6) and one ATP synthase gene (atp8). Because of the different locations of $\operatorname{trn} H$ gene, positions of the start codon of nad5 and nad6 genes and the end codon of nad4 and nad6 genes had been changed. Comparing the region (from nad5 to nad6) of mitochondrial genomes of SBPH with that of previous study, we found the differences were $57 \mathrm{bp}$ deletion in the front of nad5 gene, $21 \mathrm{bp}$ deletion at the end of nad4 gene and $84 \mathrm{bp}$ insert in the front and 55 bp deletion at the end of nad 6 gene. Furthermore, there also existed $21 \mathrm{bp}$ absent in the nad 2 gene and $60 \mathrm{bp}$ absent at the end of atp 8 gene (Additional file 9: Table S6).

Excluding five length distinct genes (nad2, nad4, nad5, nad6 and atp8) of mitochondrial genome of these two $\mathrm{SBPH}$, the other eight PCGs and two ribosomal RNA genes represented significant difference in the evolution rates (Additional file 10: Figure S4). The nad4L gene which was located in the minority strand (N-strand) showed the fastest mutation rate among these genes. The mutation rates of the other PCGs ranged from 0.0022 to 0.0076 . The evolution rate of $r r n S$ gene $(0.8 \%)$ 


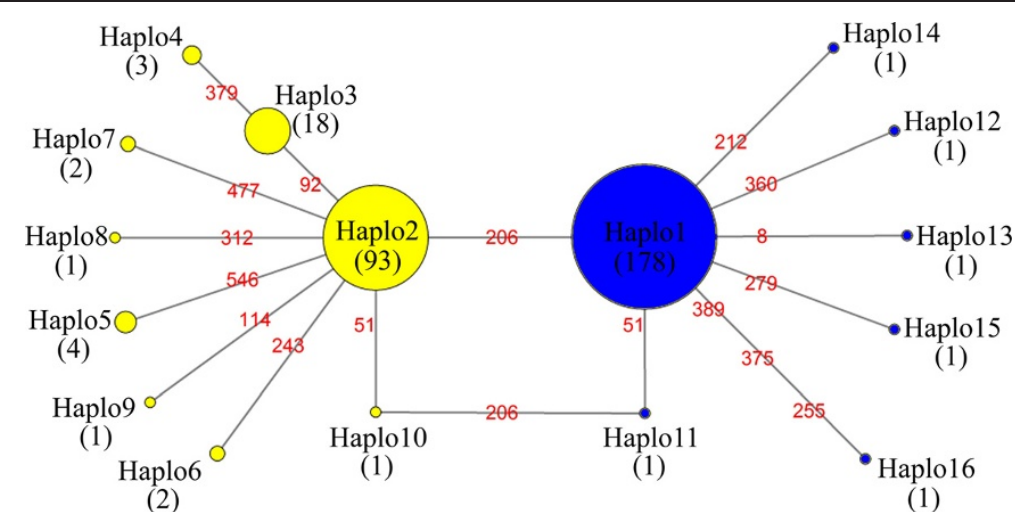

Figure 7 Haplotype network based on a fragment of Laodelphax striatellus mitochondrial sequence (642 bp) composing of nad5 (482 bp), trnH (62 bp), 2 intergenic nucleotides and nad4 genes (96 bp). Frequency of each haplotype is proportional to circle area and indicated by the numbers. The numbers on the line between circles represents the positions of each mutational site in the sequence (see more variable nucleotide site information in Additional file 8: Table S5).

was five times faster than $r r n L$ gene (0.16\%). In general, except the $\operatorname{trn} H$ and two trnS genes, the other tRNA genes were conserved (only few of them have single variant site).

Indeed, many insecticide resistances in arthropod pests correlated with genotype of mitochondria [2,49-52] and membrane proteins of mitochondria [53] had been reported. It is not clear whether variations found in mitochondria of planthoppers are directly implicated in resistance. Furthermore, the phenomenon that insecticide selection on heteroplasmic individuals favors those carrying resistant haplotypes in natural population had been revealed in Tetranychus urticae [2]. Whether similar mechanism referring to very rapid evolution and mutations being fixed occurred in planthoppers was also unknown. These aspects are well worth further study.

\section{Conclusion}

The rearrangement of mitochondrial genome of planthoppers reported in this study adds more examples of the rearranged pattern of genes in the mitochondrial genomes of hemipteran insects studied so far. The fact that the rearrangement was conserved in Delphacidae suggests that gene rearrangements of mitochondrial genome probably occurred before the differentiation of this family. Compared with other hemipterans and other insects, the extreme short lengths of atp 8 genes found in BPH and $\mathrm{SBPH}$ and the additional repeats of $\operatorname{trn} C$ found in $\mathrm{BPH}$ raise the possibility of functional changes of these genes. The variable number of tandem repeats (VNTRs) detected in the control region might provide additional genetic markers for studying population genetics, individual difference and phylogeographics of planthoppers. The comparative analyses of two sequenced mitochondrial genomes of $\mathrm{SBPH}$ revealed that rearrangements of genes in the mitochondrial genome could have occurred in the same species. On account of the different locations of $t r n H$ gene, the start and end codon of its neighboring genes had been changed. Even the same tRNA gene (e.g. trnS1 (AGN) and trnS2 (UCN) genes) could form different secondary structures in these two different individuals of SBPH. All these characteristics reveal that great divergence of mitochondrial genome could occur in different individuals of a species.

\section{Methods}

\section{Sample origin and DNA sequence assembly}

Lab-reared populations of Laodelphax striatellus (Nanjing, Jiangsu province, June 2010) and Nilaparvata lugens (Sanya, Hainan province, August 2010) for mitochondrial genome sequence were collected in China. Other natural populations which were used to validating location of $\operatorname{trnH}$ of SBPH and detecting the variable number of tandem repeats (VNTRs) in control regions of $\mathrm{BPH}$ and SBPH were collected in different locations of China (Additional file 11: Table S7). Following sequencing the genome of Wolbachia infected in these two planthopper species, partial mtDNAs of the two planthoppers were also generated using Illumina HiSeq $^{\text {tw }} 2000$. From the pair-end reads (read length: 90 bp) generated from the PCR-free random shotgun-sequencing libraries of average insert size $500 \mathrm{bp}$, one scaffold composed of three contigs of mitochondrial genome of SBPH (93,375 reads with approximately 520-fold coverage) and one scaffold composed of four contigs of mitochondrial genome of BPH (71,280 reads with approximately 400-fold coverage) were generated (Figure 1).

\section{Gap closing-PCR amplification, cloning and sequencing}

According to the flanking sequences of the gaps assembled from the filtered data, we designed 11 pairs of 
perfectly matched primers to connect the adjacent contigs (Additional file 7: Table S4). In order to reduce the error probability, we chose the high fidelity DNA polymerase Phusion ${ }^{\odot}$ High-Fidelity DNA Polymerase (New England Biolabs) to perform the PCR amplification. The cycling conditions: $98^{\circ} \mathrm{C} 30$ seconds, followed by 35 cycles of $10 \mathrm{~s}$ at $98^{\circ} \mathrm{C}, 30 \mathrm{~s}$ at different annealing temperatures, 1-2.5 min at $72^{\circ} \mathrm{C}$ depending on the size of amplicons, and the subsequent final elongation step at $72^{\circ} \mathrm{C}$ for 10 mins. The quality of PCR products was examined by $1 \%$ agarose gel electrophoresis. After purification, PCR products which were shorter than $800 \mathrm{bp}$ were directly sequenced from both directions using the ABI 3730XL Genetic Analyzer (PE Applied Biosystems). Otherwise, the PCR fragments (>800 bp) were ligated into pEASY-Blunt cloning vector (Beijing TransGen Biotech) and resulting plasmid DNAs were isolated and used for sequencing. For each larger PCR product, at least two independent clones were sequenced to ensure that we obtained the consistent sequence. The flanking sequences of each gap were exactly matched with the sequences generated based on PCR method. For closing the gaps which contain the AT-rich region and detecting the variable number of tandem repeats (VNTRs) in natural population individuals, we chose the PrimeSTAR GXL DNA polymerase (TAKARA) to perform the PCR amplification. The complete sequences of mitochondrial genome of $\mathrm{SBPH}$ and $\mathrm{BPH}$ are deposited in the GenBank database under the Accession No. JX880068, JX880069 respectively.

\section{Annotation and analysis}

After finished the gap closing work, we annotated the mitochondrial genomes of $\mathrm{BPH}$ and SBPH. All PCGs were identified by ORF Finder implemented at the NCBI website with the invertebrate mitochondrial genetic codes. Finally pair-wise comparisons with orthologous proteins from other hemipteran insects were performed to better define the limits of PCGs. The transfer RNA (tRNA) genes were identified using both of the tRNAscanSE [54] and ARWEN [55] programs and recognized manually as sequences having the appropriate anticodon and capable of folding into the typical cloverleaf secondary structure. The locations of the two rRNA genes $(r r n L$ and $r r n S$ ) were determined based on alignments and secondary structures of rRNA sequences of other hemipteran species. The boundaries of the ribosomal $r r n L$ gene were assumed to be delimited by the ends of the $\operatorname{trnL1}$ (CUN) and $\operatorname{trn} V$. The $3^{\prime}$ end of $r r n S$ gene was assumed to be delimited by the start of $\operatorname{trn} V$ while the $5^{\prime}$ end was determined through comparison with orthologous genes of other hemipteran species sequenced so far. The base composition, the Relative Synonymous Codon Usage (RSCU) values of BPH and SBPH were calculated with MEGA 4 program [56].

\section{Comparative genomics analysis}

After downloaded other mitochondrioal genome sequence (Heteroptera, 31 species; Sternorrhyncha, 9 species; Auchenorrhyncha, 7 species, Additional file 5: Table S3), we compared the two planthopper species with other hemipteran species, including the gene order, the base composition and the GC/AT-skew. The GC-skew $=(\mathrm{G}-\mathrm{C}) /(\mathrm{G}+\mathrm{C})$ and AT-skew $=(\mathrm{A}-\mathrm{T}) /(\mathrm{A}+\mathrm{T})$ were used [57] to measure the base compositional difference between different taxa in Hemiptera. In addition, in order to reveal whether two trn $H$ rearrangement types really exist in natural populations, we sequenced a region of mitochondrial genome encompassing the trnH gene from 309 individuals of $\mathrm{SBPH}$ which were collected from 15 natural populations and also checked the Wolbachia infection status of each individual (Additional file 11: Table S7). After got the sequences, we used Network 4.610 (www.fluxus-engineering. com) to calculate the frequency of each haplotype of the sequence of this region. The sequences of each haplotype are deposited in the GenBank database under the Accession No. KC006945 - KC006960.

\section{Additional files}

\begin{abstract}
Additional file 1: Table S1. Annotation for the mitochondrial genome of Nilaparvata lugens.

Additional file 2: Figure S1. Putative secondary structures of the 22 tRNA genes identified in the mitochondrial genome of Nilaparvata lugens. All tRNA genes are shown in the order of occurrence in the mitochondrial genome starting from trnl. Bars indicate Watson-Crick base pairings, and dots between $\mathrm{G}$ and $U$ pairs mark canonical base pairings appearing in tRNA.

Additional file 3: Figure S2. Putative secondary structures of the 22 tRNA genes identified in the mitochondrial genome of Laodelphax striatellus. All tRNA genes are shown in the order of occurrence in the mitochondrial genome starting from trnl. Bars indicate Watson-Crick base pairings, and dots between $\mathrm{G}$ and $U$ pairs mark canonical base pairings appearing in tRNA.
\end{abstract}

Additional file 4: Table S2. Annotation for the mitochondrial genome of Laodelphax striatellus.

Additional file 5: Table S3. Information concerning the hemipteran species with complete or nearly complete mitochondrial genome used in this study.

Additional file 6: Figure S3. Row correlation of the $A+T$ content values between the 13 PCGs, rrnL gene and rrns gene and the entire mitochondrial genome in all hemipteran insects sequenced so far.

Additional file 7: Table S4. The primers used for the PCR analysis and gap closing of two mitochondrial genomes in the present study.

Additional file 8: Table S5. Variable nucleotide site information of the "nad5-trnH-nad4" region in the mitochondrial genome of Laodelphax striatellus.

Additional file 9: Table S6. Comparison and re-annotation of previously studied mitochondrial genomes of Laodelphax striatellus.

Additional file 10: Figure S4. The mutation rates of each gene between mitochondrial genomes of two individuals of Laodelphax striatellus. The other PCGs (nad2, atp8, nad5, nad4 and nad6) were not calculated for the variant length of these genes in these two individual mitochondria. 
Additional file 11: Table S7. Collection information for natural populations of Nilaparvata lugens and Laodelphax striatellus used in this study.

\section{Competing interests}

The authors declare that they have no competing interests.

\section{Authors' contributions}

KZ conceived and designed the experiments, carried out the data analyses and drafted the manuscript. $\mathrm{XH}$ directed and coordinated this study and revised the manuscript. KZ, WZ, XR and YZ carried out molecular experiments. WZ, XD, JL and DC collected the samples and helped with DNA extraction. YD helped with the sequence reads assembly. All authors read and approved the final manuscript.

\section{Acknowledgements}

We thank Dr. Ming-Long Yuan (Lanzhou University, Lanzhou, China) for helpful comments on the manuscript. We thank Dr. Hu Li (Department of Entomology, China Agricultural University, Beijing, China) for valuable communication and advice in statistical analysis. This study was supported in part by grants from the Special Fund for Agro-Scientific Research in the Public Interest (Nos. 200903051; 200803003) from the Ministry of Agriculture of China, and a grant-in-aid from the Specialized Research Fund for the Doctoral Program of Higher Education (SRFDP) from the Ministry of Education of China (Priority Development Area, No. 20110097130005).

\section{Author details}

'Department of Entomology, Nanjing Agricultural University, Nanjing 210095, China. ${ }^{2}$ Beijing Genomics Institute, Shenzhen 518083, China.

Received: 3 December 2012 Accepted: 19 June 2013

Published: 22 June 2013

\section{References}

1. Boore JL: Animal mitochondrial genomes. Nucleic Acids Res 1999 27(8):1767-1780.

2. Van Leeuwen $T$, Vanholme B, Van Pottelberge $S$, Van Nieuwenhuyse $P$, Nauen R, Tirry L, Denholm I: Mitochondrial heteroplasmy and the evolution of insecticide resistance: non-Mendelian inheritance in action. Proc Natl Acad Sci USA 2008, 105(16):5980-5985.

3. Boore JL, Lavrov DV, Brown WM: Gene translocation links insects and crustaceans. Nature 1998, 392(6677):667-668.

4. Boore JL, Brown WM: Big trees from little genomes: mitochondrial gene order as a phylogenetic tool. Curr Opin Genet Dev 1998, 8(6):668-674

5. Boore JL: The use of genome-level characters for phylogenetic reconstruction. Trends Ecol Evol 2006, 21(8):439-446.

6. Dowton M, Castro LR, Austin AD: Mitochondrial gene rearrangements as phylogenetic characters in the invertebrates: the examination of genome 'morphology'. Invertebr Syst 2002, 16(3):345-356.

7. Dowton M, Austin AD: Evolutionary dynamics of a mitochondrial rearrangement "hot spot" in the hymenoptera. Mol Biol Evol 1999, 16(2):298-309.

8. Wei SJ, Shi M, Chen XX, Sharkey MJ, van Achterberg C, Ye GY, He JH: New views on strand asymmetry in insect mitochondrial genomes. PLOS One 2010, 5(9):e12708.

9. Zhang DX, Szymura JM, Hewitt GM: Evolution and structural conservation of the control region of insect mitochondrial DNA. J Mol Evol 1995, 40(4):382-391

10. Shao RF, Kirkness EF, Barker SC: The single mitochondrial chromosome typical of animals has evolved into 18 minichromosomes in the human body louse, Pediculus humanus. Genome Res 2009, 19(5):904-912.

11. Cameron SL, Yoshizawa K, Mizukoshi A, Whiting MF, Johnson KP: Mitochondrial genome deletions and minicircles are common in lice (Insecta: Phthiraptera). BMC Genomics 2011, 12:394.

12. Schuh RT, Slater JA: True bugs of the world (Hemiptera: Heteroptera): classification and natural history. New York, the United States of America: Cornell University Press; 1995.

13. Carver M, Gross G, Woodward T: Hemiptera (bugs, leafhoppers, cicadas, aphids, scale insects etc.), The Insects of Australia: a Textbook for Students and Research Workers. vol. 1. 2nd edition. Melbourne, Australia: Melbourne University Press; 1991:429-509.
14. Song N, Liang AP: Complete mitochondrial genome of the small brown planthopper, Laodelphax striatellus (Delphacidae: Hemiptera), with a novel gene order. Zool Sci 2009, 26(12):851-860

15. Cryan JR, Urban JM: Higher-level phylogeny of the insect order Hemiptera: is Auchenorrhyncha really paraphyletic? Syst Entomol 2012, 37:7-21.

16. Song N, Liang AP, Ma C: The complete mitochondrial genome sequence of the planthopper, Sivaloka damnosus. J Insect Sci 2010, 10:76.

17. Song N, Liang A: The complete mitochondrial genome sequence of Geisha distinctissima (Hemiptera: Flatidae) and comparison with other hemipteran insects. Acta Biochim Biophys Sin (Shanghai) 2009, 41(3):206-216

18. Lee W, Kang J, Jung C, Hoelmer K, Lee SH, Lee S: Complete mitochondrial genome of brown marmorated stink bug Halyomorpha halys (Hemiptera: Pentatomidae), and phylogenetic relationships of hemipteran suborders. Mol Cells 2009, 28(3):155-165.

19. Talavera G, Vila R: What is the phylogenetic signal limit from mitogenomes? The reconciliation between mitochondrial and nuclear data in the Insecta class phylogeny. BMC Evol Biol 2011, 11(1):315.

20. Hibino H: Biology and epidemiology of rice viruses. Annu Rev Phytopathol 1996, 34:249-274.

21. Cheng J: Rice planthopper problems and relevant causes in China In Planthoppers: New Threats to the Sustainability of Intensive Rice Production Systems in Asia. Edited by Heong KL, Hardy B. Los Baños, Philippines: International Rice Research Institute; 2009:157-178.

22. Hilgenboecker $K$, Hammerstein P, Schlattmann P, Telschow A, Werren JH: How many species are infected with Wolbachia?-A statistical analysis of current data. FEMS Microbiol Lett 2008, 281(2):215-220.

23. Zhang $\mathrm{H}$, Zhang $\mathrm{K}$, Hong $\mathrm{XY}$ : Population dynamics of noncytoplasmic incompatibility-inducing Wolbachia in Nilaparvata lugens and its effects on host adult life span and female fitness. Environ Entomol 2010, 39(6):1801-1809.

24. Noda H, Koizumi Y, Zhang Q, Deng K: Infection density of Wolbachia and incompatibility level in two planthopper species, Laodelphax striatellus and Sogatella furcifera. Insect Biochem Mol Biol 2001, 31(6-7):727-737.

25. Shao R, Mitani H, Barker SC, Takahashi M, Fukunaga M: Novel mitochondrial gene content and gene arrangement indicate illegitimate inter-mtDNA recombination in the chigger mite, Leptotrombidium pallidum. $J \mathrm{Mol}$ Evol 2005, 60(6):764-773.

26. Jeyaprakash A, Hoy MA: The mitochondrial genome of the predatory mite Metaseiulus occidentalis (Arthropoda: Chelicerata: Acari: Phytoseiidae) is unexpectedly large and contains several novel features. Gene 2007, 391(1-2):264-274

27. Hua JM, Li M, Dong PZ, Cui Y, Xie Q, Bu WJ: Phylogenetic analysis of the true water bugs (Insecta: Hemiptera: Heteroptera: Nepomorpha): evidence from mitochondrial genomes. BMC Evol Biol 2009, 9:134

28. Li H, Liu H, Shi A, Stys P, Zhou X, Cai W: The complete mitochondrial genome and novel gene arrangement of the unique-headed bug Stenopirates sp. (Hemiptera: Enicocephalidae). PLoS One 2012, 7(1):e29419.

29. Li H, Gao JY, Liu HY, Liu H, Liang AP, Zhou XG, Cai WZ: The architecture and complete sequence of mitochondrial genome of an assassin bug Agriosphodrus Dohrni (Hemiptera: Reduviidae). Int J Biol Sci 2011 7(6):792-804.

30. Li H, Liu HY, Cao LM, Shi AM, Yang HL, Cai WZ: The complete mitochondrial genome of the damsel bug Alloeorhynchus bakeri (Hemiptera: Nabidae). Int J Biol Sci 2012, 8(1):93-107.

31. Lavrov DV, Brown WM, Boore J: A novel type of RNA editing occurs in the mitochondrial tRNAs of the centipede Lithobius forficatus. Proc Natl Acad Sci USA 2000, 97(25):13738-13742.

32. Hua J, Li M, Dong P, Cui Y, Xie Q, Bu W: Comparative and phylogenomic studies on the mitochondrial genomes of Pentatomomorpha (Insecta: Hemiptera: Heteroptera). BMC Genomics 2008, 9:610.

33. Thao ML, Baumann $L$, Baumann P: Organization of the mitochondrial genomes of whiteflies, aphids, and psyllids (Hemiptera, Sternorrhyncha). BMC Evol Biol 2004, 4:25.

34. Salvato P, Simonato M, Battisti A, Negrisolo E. The complete mitochondrial genome of the bag-shelter moth Ochrogaster lunifer (Lepidoptera, Notodontidae). BMC Genomics 2008, 9:331.

35. Zhu YJ, Zhou GL, Fang R, Ye J, Yi JP: The complete sequence determination and analysis of Lymantria dispar (Lepidoptera: Lymantriidae) mitochondrial genome. Plant Quarantine 2010, 24(4):6-11. 
36. Sharp PM, Tuohy TM, Mosurski KR: Codon usage in yeast: cluster analysis clearly differentiates highly and lowly expressed genes. Nucleic Acids Res 1986, 14(13):5125-5143.

37. Dotson $E$, Beard CB: Sequence and organization of the mitochondrial genome of the Chagas disease vector, Triatoma dimidiata. Insect Mol Biol 2001, 10(3):205-215.

38. Zhang DX, Hewitt GM: Insect mitochondrial control region: a review of its structure, evolution and usefulness in evolutionary studies. Biochem Syst Ecol 1997, 25(2):99-120.

39. Cameron SL, Beckenbach AT, Dowton M, Whiting M: Evidence from mitochondrial genomics on interordinal relationships in insects. Arthropod Syst Phylogeny 2006, 64(1):27-34.

40. Cameron SL, Lambkin CL, Barker SC, Whiting MF: A mitochondrial genome phylogeny of Diptera: whole genome sequence data accurately resolve relationships over broad timescales with high precision. Syst Entomol 2006, 32(1):40-59.

41. Cameron SL, Dowton M, Castro LR, Ruberu K, Whiting MF, Austin AD, Diement K, Stevens J: Mitochondrial genome organization and phylogeny of two vespid wasps. Genome 2008, 51(10):800-808

42. Fenn JD, Song H, Cameron SL, Whiting MF: A preliminary mitochondrial genome phylogeny of Orthoptera (Insecta) and approaches to maximizing phylogenetic signal found within mitochondrial genome data. Mol Phylogenet Evol 2008, 49(1):59-68.

43. Sheffield NC, Song H, Cameron SL, Whiting MF: Nonstationary evolution and compositional heterogeneity in beetle mitochondrial phylogenomics. Syst Biol 2009, 58(4):381-394

44. Baudry E, Bartos J, Emerson K, Whitworth T, Werren J: Wolbachia and genetic variability in the birdnest blowfly Protocalliphora sialia. Mol Ecol 2003, 12(7):1843-1854.

45. Dean MD, Ballard KJ, Glass A, Ballard JW: Influence of two Wolbachia strains on population structure of East African Drosophila simulans. Genetics 2003, 165(4):1959-1969.

46. Jiggins FM: Male-killing Wolbachia and mitochondrial DNA: selective sweeps, hybrid introgression and parasite population dynamics. Genetics 2003, 164(1):5-12.

47. Shoemaker DD, Keller G, Ross KG: Effects of Wolbachia on mtDNA variation in two fire ant species. Mol Ecol 2003, 12(7):1757-1771.

48. Shoemaker DD, Dyer KA, Ahrens M, McAbee K, Jaenike J: Decreased diversity but increased substitution rate in host mtDNA as a consequence of Wolbachia endosymbiont infection. Genetics 2004, 168(4):2049-2058.

49. Kadir HA, Knowles CO: Inhibition of ATP dephosphorylation by acaricides with emphasis on the anti-ATPase activity of the carbodiimide metabolite of diafenthiuron. J Econ Entomol 1991, 84(3):801-805.

50. Dekeyser MA: Acaricide mode of action. Pest Manag Sci 2004, 61(2):103-110

51. Song C, Scharf ME: Mitochondrial impacts of insecticidal formate esters in insecticide-resistant and insecticide-susceptible Drosophila melanogaster. Pest Manag Sci 2009, 65(6):697-703.

52. Van Pottelberge $S$, Van Leeuwen $T$, Nauen R, Tirry L: Resistance mechanisms to mitochondrial electron transport inhibitors in a field-collected strain of Tetranychus urticae Koch (Acari: Tetranychidae). Bull Entomol Res 2009, 99(1):23-31.

53. Guzov VM, Unnithan GC, Chernogolov AA, Feyereisen R: CYP12A1, a mitochondrial cytochrome P450 from the house fly. Arch Biochem Biophys 1998, 359(2):231-240.

54. Lowe TM, Eddy SR: tRNAscan-SE: a program for improved detection of transfer RNA genes in genomic sequence. Nucleic Acids Res 1997, 25(5):955-964

55. Laslett D, Canback B: ARWEN: a program to detect tRNA genes in metazoan mitochondrial nucleotide sequences. Bioinformatics 2008, 24(2):172-175
56. Tamura K, Dudley J, Nei M, Kumar S: MEGA4: Molecular Evolutionary Genetics Analysis (MEGA) software version 4.0. Mol Biol Evol 2007, 24(8):1596-1599.

57. Perna NT, Kocher TD: Patterns of nucleotide composition at fourfold degenerate sites of animal mitochondrial genomes. J Mol Evol 1995, 41(3):353-358.

doi:10.1186/1471-2164-14-417

Cite this article as: Zhang et al:: The complete mitochondrial genomes of two rice planthoppers, Nilaparvata lugens and Laodelphax striatellus: conserved genome rearrangement in Delphacidae and discovery of new characteristics of atp8 and tRNA genes. BMC Genomics 2013 14:417.

\section{Submit your next manuscript to BioMed Central and take full advantage of:}

- Convenient online submission

- Thorough peer review

- No space constraints or color figure charges

- Immediate publication on acceptance

- Inclusion in PubMed, CAS, Scopus and Google Scholar

- Research which is freely available for redistribution

Submit your manuscript at www.biomedcentral.com/submit
C) Biomed Central 